\title{
Erratum: Characteristic functions of quantum heat with baths at different temperatures [Phys. Rev. E 97, 062117 (2018)]
}

\author{
Erik Aurell 1
}

(Received 18 September 2019; published 27 September 2019)

DOI: 10.1103/PhysRevE.100.039902

\section{THE CORRECTION}

In this paper, in Eqs. (26) and (A14), a factor in the quantity called $\mathcal{J}^{(2)}$ was incorrectly given as $\frac{y^{\prime} z^{\prime}-y z}{\Delta}-\frac{1}{2}$. It should read $\frac{y z^{\prime}-y^{\prime} z}{\Delta}-\frac{1}{2}$. Although this error does not change the value or the first derivative of the generating function at $v=0$, it changes higher moments. The second derivative of the generating function at $v=0$ was, hence, incorrectly given in this paper in Eq. (A23); the correct expression should have 1 inside the square brackets, instead of $1+\operatorname{coth}^{2} \frac{\omega \hbar \beta}{2}$.

\section{CONSEQUENCES OF THE CORRECTION}

As treated in the original paper, the generating function of the energy change in the bath is the functional,

$$
G_{i f}(v)=\operatorname{Tr}_{B}\left\langle f\left|e^{i \nu H_{B}} U e^{-i \nu H_{B}}\left[|i\rangle\langle i| \otimes \rho_{B}(\beta)\right] U^{\dagger}\right| f\right\rangle,
$$

where $i$ and $f$ are the initial and the final states of the system, $\rho_{B}(\beta)$ is the initial thermal state of the bath at inverse temperature $\beta$, and $v$ is the generating function parameter. In the Feynman-Vernon approach, the two unitary operators and the initial thermal state of the bath in (1) are expressed as path integrals, and the histories of the bath then integrated out. For baths that are harmonic oscillators, this gives a result which, in principle, must look like

$$
G_{i f}(v)=\int_{i f} \mathcal{D} X \mathcal{D} Y \exp \left\{\frac{i}{\hbar} S_{S}[X]-\frac{i}{\hbar} S_{S}[Y]+\frac{i}{\hbar} S_{i}^{(v)}[X, Y]-\frac{1}{\hbar} S_{r}^{(v)}[X, Y]\right\},
$$

where $S_{i}^{(\nu)}[X, Y]$ and $S_{r}^{(\nu)}[X, Y]$ are quadratic functions of the forward and backward system paths $(X$ and $Y)$ and where $\int_{i f}(\cdots)$ is a shorthand for projections on initial and final states. After the correction, the functionals in (2) can be written

$$
\begin{aligned}
\frac{i}{\hbar} S_{i}^{(v)}-\frac{1}{\hbar} S_{r}^{(v)}= & \frac{i}{\hbar} \int^{t} \int^{s}\left(X X^{\prime}-Y Y^{\prime}\right) k_{i}\left(s, s^{\prime}\right) d s^{\prime} d s-\frac{1}{\hbar} \int^{t} \int^{s}\left(X X^{\prime}+Y Y^{\prime}\right) k_{r}\left(s, s^{\prime}\right) d s^{\prime} d s \\
& +\frac{i}{\hbar} \int^{t} \int^{t} X Y^{\prime} k_{i}^{(v)}\left(s, s^{\prime}\right) d s^{\prime} d s+\frac{1}{\hbar} \int^{t} \int^{t} X Y^{\prime} k_{r}^{(v)}\left(s, s^{\prime}\right) d s^{\prime} d s,
\end{aligned}
$$

where primed (unprimed) quantities refer to time $s^{\prime}(s)$, the four kernels are

$$
\begin{array}{ll}
k_{i}\left(s, s^{\prime}\right)=\sum_{b} \frac{C_{b} C_{b}^{\prime}}{2 m_{b} \omega_{b}} \sin \omega_{b}\left(s-s^{\prime}\right) & k_{i}^{(v)}\left(s, s^{\prime}\right)=\sum_{b} \frac{C_{b} C_{b}^{\prime}}{2 m_{b} \omega_{b}} \sin \omega_{b}\left(s-s^{\prime}+\hbar \nu\right) \\
k_{r}\left(s, s^{\prime}\right)=\sum_{b} \frac{C_{b} C_{b}^{\prime}}{2 m_{b} \omega_{b}} \operatorname{coth}\left(\frac{\omega \hbar \beta}{2}\right) \cos \omega_{b}\left(s-s^{\prime}\right) & k_{r}^{(\nu)}\left(s, s^{\prime}\right)=\sum_{b} \frac{C_{b} C_{b}^{\prime}}{2 m_{b} \omega_{b}} \operatorname{coth}\left(\frac{\omega \hbar \beta}{2}\right) \cos \omega_{b}\left(s-s^{\prime}+\hbar \nu\right)
\end{array}
$$

and where $C_{b}$ 's are the possibly time-dependent interaction coefficients among the system and oscillator $b$ and $m_{b}$ and $\omega_{b}$ are the mass and angular frequency of that oscillator. More details of the derivation of (3) in the path integral formulation can be found on-line [1]. Another derivation of (3) not relying on path integrals can be found in Ref. [2].

[1] E. Aurell, A simplified characteristic functions of quantum heat, arXiv:1904.03496.

[2] E. Aurell, R. Kawai, and K. Goyal, An operator derivation of the Feynman-Vernon theory, with applications to the generating function of bath energy changes and to anharmonic baths, arXiv:1907.02671. 